\title{
A Review of MicroRNAs Related to the Occurrence, Diagnosis, and Prognosis of Non-small Cell Lung Cancer
}

\author{
Jun Wanga, Li Ke ${ }^{\mathrm{a}^{*}}$, Xian-liang Jianga, Can Zhang \\ ${ }^{a}$ Department of Chest Surgery, Anhui Provincial Hospital, Hefei 230001, Anhui, China.
}

\section{Abstract}

Non-small cell lung cancer (NSCLC) is the main most common type of lung cancer, accounting for about 80\% of all cases. The five-year survival rate of patients with NSCLC is usually less than 20\%. MicroRNAs (miRNAs), a type of small non-coding RNA, are closely related to the development and occurrence of tumors, including NSCLC. Here, we reviewed the miRNAs related to the occurrence, diagnosis and prognosis of NSCLC.

Keywords: miRNA; non-small cell lung cancer; occurrence; diagnosis; prognosis

\section{INTRODUCTION}

Lung cancer is a malignant tumor with the highest morbidity and mortality rates in the world. According to the International Agency for Research on Cancer ${ }^{[1]}$, 1.82 million new cases of lung cancer were diagnosed worldwide in 2012, ranking first among all malignant tumors; the incidence and mortality rates of lung cancer in China are similarly alarming ${ }^{[2,3]}$. Non-small cell lung cancer (NSCLC) is the most common type of lung cancer, accounting for about $80 \%$ of all lung cancers ${ }^{[4]}$. NSCLC is mainly divided into squamous cell carcinoma, adenocarcinoma, adenosquamous carcinoma, largecell lung cancer, and sarcomatoid carcinoma. Due to the lack of obvious clinical manifestations in its early stages, NSCLC is typically discovered late in the disease process; as a result, the prognosis of NSCLC is poor, and the five-year survival rate is usually less than $20 \%$ ${ }^{[5]}$. Therefore, studying the mechanism of NSCLC occurrence and development is crucial for the diagnosis and treatment of NSCLC.

MicroRNA (miRNA) is a type of small molecule noncoding RNA with a length of about 18-25 base pairs, which regulate gene expression after transcription. It has been reported that miRNAs are abnormally expressed in a variety of tumors, and the dysregulated miRNAs may serve as tumor promoters or inhibitors ${ }^{[6]}$. MiRNAs can regulate cell proliferation, differentiation and apoptosis through complete or incomplete match-

*Corresponding author: Li Ke

Mailing address: Department of Chest Surgery, Anhui Provincial Hospital. No.17 Lujiang Road, Hefei 230001, Anhui, People's Republic of China.

E-mail: keli_kl@163.com

Received: 29 July 2018 Accepted: 20 September 2018 ing with the target gene mRNA 3'UTR; thus they play an important role in the development of tumors and in tumor therapy research ${ }^{[7]}$. Studies have shown that miRNAs have specific expression profiles in NSCLC, and serve as oncogenes or tumor-suppressor genes to promote or inhibit NSCLC cell proliferation, migration, and invasion ${ }^{[8]}$. In addition, miRNAs can be used for the early detection, prognosis, radio-sensitivity and chemo-sensitivity prediction of NSCLC ${ }^{[9]}$.

In this review, we summarized the miRNAs associated with carcinogenesis and cancer suppression in NSCLC and emphasized miRNAs' potential as biomarkers for clinical diagnosis, prognosis and treatment.

\section{MIRNAS IN THE OCCURRENCE AND DEVELOPMENT OF NSCLC}

With the development of molecular biology technology, miRNA has gradually become a research hotspot, which provides a new direction for tumor research. Many studies have shown that miRNAs can serve as oncogenes or tumor suppressor genes to affect their target genes and signaling pathway activities, playing an important role in the occurrence and development of NSCLC ${ }^{[10,11]}$.

\section{MiRNAs as oncogenes in NSCLC}

It has been demonstrated that miR-221 is aberrantly up-regulated in NSCLC tissues and NSCLC cells compared to normal tissues and cells, and has an oncogenic role by directly targeting TIMP2 ${ }^{[12]}$. MiR-1285-5p promotes the proliferation, migration and invasion of NSCLC cells via down-regulating the two genes, Smad4 and $\mathrm{CDH} 1{ }^{[13]}$. In addition, Wang et al. ${ }^{[14]}$ discovered that miR-661 could target RUNX3 and serve as an oncogene in NSCLC. Liu et al. ${ }^{[15]}$ also revealed that miR- 
661 was up-regulated in NSCLC tissues compared with the paired adjacent tissues, and miR-661 promoted tumor invasion and metastasis by directly inhibiting RB1 in NSCLC. Huang et al. ${ }^{[16]}$ showed that miR-10b expression levels were higher in NSCLC tissues compared with an adjacent normal tissue control, and it could promote cancer cell progress by accelerating NSCLC's cell cycle progression in the G0/G1 phase and suppressing cell apoptosis. Apaf- 1 is associated with the suppression of apoptosis. In NSCLC cells, miR-484 accelerates the cell progression by inhibiting Apaf1 expression ${ }^{[17]}$. MiR-19 can enhance proliferation and inhibit apoptosis via the EGFR signaling pathway by targeting PP2A and BIM in NSCLC ${ }^{[18]}$. In addition, Zeng et al. ${ }^{[19]}$ demonstrated that miR-205 promoted NSCLC cell growth in vitro and in vivo, which was related to Smad4. Sun et al. ${ }^{[20]}$ reported that miR-346 contributed to NSCLC growth and invasion in vitro and in vivo by regulating the XPC/ERK/Snail/E-cadherin pathway. MiR-1297 functions as a tumor promoter in NSCLC cells by targeting the PTEN/Akt/Skp2 signaling pathway ${ }^{[21]}$. MiR-106a also promotes the growth and metastasis of NSCLC by targeting PTEN ${ }^{[22]}$. These oncogenic miRNAs and their targets in NSCLC are summarized in Table 1. According to the previously mentioned, we expect that when miRNA is highly expressed in NSCLC, it can play a positive role in promoting the occurrence and development of a tumor by inhibiting the expression of tumor suppressors, thus playing an oncogene role.

MiRNAs as tumor-suppressor genes in NSCLC

Apart from their roles as tumor promoters, some miRNAs function as tumor suppressors by targeting oncogenes. In NSCLC tissues and cell lines, miR-376a was significantly downregulated; the overexpression of miR-376a could inhibit cell proliferation and inva- sion and alsp increase cell apoptosis by decreasing the mRNA and protein levels of c-Myc ${ }^{[23]}$. MiRNA-183 has been considered a tumor suppressor, which can inhibit the proliferation, migration and invasion of NSCLC cells by targeting MTA1 ${ }^{[24]}$. MiR-142-3p can inhibit NSCLC cell proliferation and induces cell apoptosis through the down-regulation of HMGB1 ${ }^{[25]}$. MiRNA-204 is significantly reduced in NSCLC tissues as compared to non-neoplastic tissues, and miRNA-204 can suppress NSCLC cell proliferation and migration, and induce apoptosis and G1 arrest through negatively regulating JAK2 ${ }^{[26]}$ or ATF2 ${ }^{[27]}$.

In addition, Zhang et al. ${ }^{[28]}$ reported that the low expression of miR-770 was correlated with poor patient survival in NSCLC, while miR-770 overexpression was capable of inhibiting NSCLC tumor growth by down-regulating JMJD6 and its downstream WNT/ $\beta$-catenin pathway both in vitro and in vivo. Chen et al. ${ }^{[29]}$ demonstrated that the mTOR signaling pathway was dysregulated in more than $50 \%$ of all human malignancies and was a major target in cancer treatment, miR145-3p inhibits cell growth, motility, and chemotaxis in NSCLC through suppression of the mTOR pathway by targeting PDK1. Zhou et al. ${ }^{[30]}$ revealed that miR135 a promoted cell apoptosis and inhibited cell proliferation, migration, invasion and tumor angiogenesis by targeting the IGF-1 gene through the IGF-1/PI3K/ Akt signaling pathway in NSCLC. Jiang et al. ${ }^{[31]}$ showed that miR-1258 could inhibit NSCLC cell proliferation and induced senescence and apoptosis via targeting the GRB2/Ras/Erk pathway. Furthermore, miR-542$3 p$ exerts tumor suppressive functions in NSCLC by down-regulating FTSJ2 ${ }^{[32]}$. MiR-509-5p decreases cell proliferation, migration, and the invasive capability of NSCLC cells by negatively regulating FOXM1 expression ${ }^{[33]}$. MiR-194 also suppresses tumor growth through function as a negative regulator of CUL4B in ESCC ${ }^{[34]}$.

Table 1 The oncogenic miRNAs in NSCLC

\begin{tabular}{lll}
\hline miRNA & Biological mechanisms & Target genes \\
\hline miR-221 & promotes cell proliferation, cell cycle, migration and invasion & TIMP2 \\
miR-1285-5p & promotes cell proliferation, migration and invasion & CDH1 and Smad4 \\
miR-661 & promotes cell proliferation and invasion & RUNX3, RB1 \\
miR-10b & promotes cancer cell progress by accelerating cell cycle progression & Klotho \\
& in the G0/G1 phase and suppresses cell apoptosis & Apaf-1 \\
miR-484 & inhibits cell apoptosis & PP2A and BIM \\
miR-19 & promotes cell proliferation and inhibits cell apoptosis & Smad4 \\
miR-205 & promotes tumor growth & XPC/ERK/Snail/E-cadherin pathway \\
miR-346 & contributes to cell growth and invasion & PTEN/Akt/Skp2 signaling pathway \\
miR-1297 & promotes cell proliferation & PTEN \\
miR-106a & promotes tumor growth and metastasis & \\
\hline
\end{tabular}


Glycolysis has been considered a feature of cancer cells; Akt is a key enzyme in the regulation of glycolysis and energy metabolism in cancer cells. It has been determined that miR-124 is able to inhibit proliferation, glycolysis, and energy metabolism by targeting AKTGLUT1/HKII in NSCLC cells ${ }^{[35]}$. VEGFs are important mediators of angiogenesis and lymphangiogenesis during tumor development ${ }^{[36]}$. MiR-128 can suppress tumorigenesis, angiogenesis and lymphangiogenesis in ESCC through down-regulating VEGF-C ${ }^{[37]}$. The aforementioned tumor-suppressor miRNAs and their targets in NSCLC are summarized in Table 2. According to these studies, when miRNA expression is significantly reduced in NSCLC, it can regulate the growth of tumors by inhibiting the related oncogenes and tumor factors. However, the relationship between miRNAs and NSCLC, especially the delicate mechanism of gene expression regulation of miRNAs, is still unclear. Studies on these issues sholud gradually clarify the mechanism of the occurrence and development of NSCLC and may also provide new methods for the early diagnosis and treatment of NSCLC.

\section{CLINICAL APPLICATIONS OF MIRNAS IN NSCLC}

Diagnostic value of miRNAs in NSCLC

At present, the sensitivity and specificity of serum tumor markers are relatively low in the clinical diagnosis of patients with NSCLC, especially in the early stage of NSCLC. It is easy to misdiagnose or miss a diagnosis, delaying the optimum time for treatment. Profiling research has revealed the potential value of miRNAs for the early detection and screening of NSCLC. For example, Świtlik et al. ${ }^{[38]}$ showed that miR-30a-5p and miR-210-3p could be used to discriminate NSCLC tissue from non-cancerous lung tissues. Geng et al. ${ }^{[39]}$ reported that five plasma miRNAs (miR-20a, miR-145, miR-21, miR-223 and miR-221) could be used as potential biomarkers in early screening for NSCLC. Zhu et al. ${ }^{[40]}$ validated that the serum levels of three miRNAs (miR-182, miR-183, and miR-210) were significantly up-regulated and the miR-126 level was markedly down-regulated in NSCLC patients, which could serve as tumor biomarkers for early diagnosis of NSCLC. Moreover, miRNAs can also function as biomarkers for the classification of NSCLC. For example, Jin et al. ${ }^{[41]}$ demonstrated that miR-181-5p, miR-30a-3p, miR-30e$3 p$, and miR-361-5p were adenocarcinoma-specific, while miR-10b-5p, miR-15b-5p, and miR-320b were SCC-specific. Molina-Pinelo et al. ${ }^{[42]}$ showed that nine miRNAs were differentially expressed in SCC versus adenocarcinoma samples. Of these, six miRNAs (miR205, miR-149, miR-422a, miR-708, miR-378, and miR375) were validated.

Furthermore, circulating miRNAs are also promising biomarkers for cancer detection. In NSCLC, a multicentric case-control study revealed that a five-miRNA panel, including miR-483-5p, miR-193a-3p, miR-25, miR-214, and miR-7 could serve as a potential biomarker for diagnosing NSCLC in patients of different races, even in the early stages of cancer. Of the five miRNAs, miR-483-5p, miR-193a-3p, and miR-25 are tumor cell growth/cycle-related miRNAs. MiR-7 is a tissue-specific miRNA, and miR-214 may be categorized as an immune response-related miRNA ${ }^{[43]}$. Giallombardo et al. ${ }^{[44]}$ showed that eight plasma exosomal miRNAs, (miR-30b, miR-30c, miR-103, miR-122, miR-

Table 2 The tumor-suppressor miRNAs in NSCLC

\begin{tabular}{lll}
\hline miRNA & Biological mechanisms & Target genes \\
\hline miR-376a & inhibits cell proliferation and invasion, increases cell apoptosis & c-Myc \\
miR-183 & inhibits cell proliferation, migration, invasion and tumor growth in nude mice & MTA1 \\
miR-142-3p & inhibits cell proliferation and induces cell apoptosis & HMGB1 \\
miRNA-204 & suppresses cell proliferation and migration, induces apoptosis and G1 arrest & JAK2, ATF2 \\
miR-770 & inhibits NSCLC tumor growth & JMJD6 \\
miR-145-3p & inhibits cell growth, motility and chemotaxis & PDK1 \\
miR-135a & promotes cell apoptosis and inhibits cell proliferation, migration, i & IGF-1 \\
& nvasion and tumor angiogenesis & \\
miR-1258 & inhibits cell proliferation, induces cell senescence and apoptosis & GRB2/Ras/Erk pathway \\
miR-542-3p & inhibits cell proliferation, migration, cell cycle, and tumor growth, & FTSJ2 \\
& induces cell apoptosis & \\
miR-509-5p & decreases cell proliferation, migration and invasion & FOXM1 \\
miR-194 & suppresses cell proliferation and migration, and tumor growth & CUL4B \\
miR-124 & inhibits proliferation, glycolysis, and energy metabolism & AKT-GLUT1/HKII \\
miR-128 & suppresses tumourigenesis, angiogenesis and lymphangiogenesis & VEGF-C \\
\hline
\end{tabular}


195, miR-203, miR-221, and miR-222) were correlated with NSCLC.

The studies described above clearly suggest that abnormal miRNAs expressions can be used as diagnostic tools in NSCLC. However, due to the differences in methodology and quality control, as well as the limited number of microRNAs and samples studied, existing studies generally lack repeatability. Therefore, better research strategies and more detailed work will be required in the near future.

\section{The prognostic value of miRNAs in NSCLC}

Recently, many studies have reported that some miRNAs can be used as prognostic markers in NSCLC. A meta-analysis provided evidence that the high expression miR-21, miR-200c, and miR-125b was negatively associated with survival in NSCLC patients, while the high expression of miR-148b, miR-365, miR-124, miR32, miR-146a, and miR-375 was significantly associated with a better prognosis ${ }^{[45]}$. Wang and his colleagues identified that 11 miRNAs, as well as their target genes and transcription factors, may be profitable in the prognostic prediction of NSCLC as biomarkers. In NSCLC blood samples, Wang et al. ${ }^{[46]}$ found that serum miR-98 expression was down-regulated in patients with NSCLC, and the decreased serum miR-98 was positively correlated with a worse TNM stage, lymph node metastasis, as well as unfavorable overall survival. Guo et al. ${ }^{[47]}$ showed that a decreased miR-204 level in plasma is associated with a poor prognosis in NSCLC patients. Wang and his coworkers ${ }^{[48]}$ revealed that high serum miR-411 expression could serve as a non-invasive prognostic biomarker for NSCLC patients. In NSCLC tissues, Li et al. ${ }^{[49]}$ report that miR146 is highly expressed in cancer tissues, which can be used to predicts the prognosis of NSCLC. Wang et al. ${ }^{[46]}$ reveal that there is an obviously down-regulated miR$148 \mathrm{~b}$ expression in NSCLC tissues, and the low miR$148 \mathrm{~b}$ expression is closely related with poor survival of NSCLC patients. Zhang et al. ${ }^{[50]}$ demonstrate that a high expression level of miR-221 is associated with the short overall survival time for NSCLC patients. Cancer-associated fibroblasts (CAFs) play a vital role in promoting tumor progression. Hepatocyte growth factor (HGF) is a protein produced by CAFs that promotes tumor growth, motility, and morphogenesis ${ }^{[51]}$. The expression of miR-200 A in matrix fibroblasts is negatively correlated with HGF expression. High miR200a and low HGF expression in stromal fibroblasts may predict a good prognosis in patients with NSCLC ${ }^{[52]}$. Further, Zhao and his colleagues ${ }^{[53]}$. reported that miRNA single-nucleotide polymorphisms (SNPs) are associated with survival in NSCLC. Their results confirmed that one SNP, rs2042253 is located in the adjacent region of miR-5197-5p, which was associated with improved survival for late-stage NSCLC patients; another SNP, rs7522956, is located in the loop sequences of the miR-4742 gene, and it was significantly associated with the survival of early stage NSCLC patients.

Several recent studies have demonstrated that exosomal miRNAs may serve as prognostic biomarkers for NSCLC. Liu and his coworkers used a qPCR array panel, and found that increased levels of exosomal miR-23b$3 p$, miR-10b-5p and miR-21-5p were independently associated with a poor overall survival rate for NSCLC. ${ }^{[54]}$ Kanaoka et al. ${ }^{[55]}$ identified that high plasma exosomal miR-451a might be a prognostic factor for the overall survival of NSCLC patients.

Taken together, these studies show the prognostic value of miRNAs in NSCLC. Nevertheless, to avoid potential biases and limitations, additional validation is still necessary in large patient populations.

MiRNAs for overcoming treatment resistance of NSCLC

Radio- and chemo-resistance hinders success in treating NSCLC. MiRNAs are considered to be resistant against radio- and chemo-therapy in certain types of cancers, including NSCLC.

\section{Radiotherapy}

Several studies have identified miRNAs as a predictive biomarker for radiation-resistance in NSCLC. Salim et al. ${ }^{[56]}$ suggested that miRNA-214 could be a putative regulator of radiation-resistance, and miRNA-214 overexpression induced radio-resistance of NSCLC cells. Ma et al. ${ }^{[57]}$ showed that miR-95 was highly expressed in recurrent NSCLC cells, so a decrease could enhance the radio-sensitivity of NSCLC. Yin and his colleagues ${ }^{[58]}$. reported that overexpression of miR-99a increased radio-sensitivity, while inhibition of miR-99a induced radio-resistance of NSCLC cell lines in vitro and in vivo, and the role was at least partially mediated by the mTOR signaling pathway. Furthermore, miR-21 expression is increased in NSCLC; when down-regulated, it can promote NSCLC cell apoptosis induced by irradiation, which indicates that miR-21 may be a potential target for overcoming the radio-resistance of NSCLC ${ }^{[59]}$. MiR-328-3p is reduced in NSCLC tissues. Up-regulation of miR-328-3p can restore the sensitivity of NSCLC cells to radio therapy, suggesting miR-328-3p may act as a radio-sensitizer for NSCLC therapy ${ }^{[60]}$. In addition, plasma miRNAs may serve as novel biomarkers for predicting the clinical response of NSCLC radiotherapy. Bioinformatical analysis and clinical verification revealed that four plasma miRNAs (miR-98-5p, miR302e, miR-495-3p, and miR-613) could be used as radio-sensitivity indicators in patients with NSCLC ${ }^{\text {[61] }}$. 


\section{Chemotherapy}

Taxanes, including paclitaxel and docetaxel, are the standard treatment for advanced NSCLC. Chen et al. ${ }^{\left[{ }^{62]}\right.}$ reported that decreased miR-27b expression was associated with docetaxel resistance of NSCLC, while up-regulation of miR-27b could inhibit NSCLC cell viability and enhance the docetaxel sensitivity of NSCLC cells through direct inhibition of EGFR expression. Ye et al. ${ }^{\left[{ }^{[3]}\right]}$ showed that overexpression of miR-186 sensitized NSCLC cells to paclitaxel, whereas inhibition of miR-186 conferred resistance in NSCLC cells. In validation, miR-186 was found to be down-regulated in NSCLC patients who were paclitaxel-resistant, and this decrease was associated with poor survival. Xu et al. ${ }^{[64]}$ revealed a differential expression of miR-30a-5p in two NSCLC cells (paclitaxel-resistant vs paclitaxel-sensitive NSCLC cells). Their results found that up-regulation of miR-30a-5p sensitized NSCLC cells to paclitaxel both in vitro and in vivo through reducing BCL-2 expression, which provided evidence of the potential utility of miR-30a-5p as a biomarker in predicting paclitaxel responsiveness in NSCLC. Gan et al. ${ }^{[65]}$ observed that overexpression of miR-339-5p enhanced the proliferation inhibition ability of Taxol in NSCLC cells, and their in vivo study demonstrated that up-regulation of miR339-5p could promote the inhibitory function of Taxol on NSCLC growth.

Cisplatin (DDP) is another classic chemotherapy agent used in the treatment of NSCLC. Yuwen et al. ${ }^{[66]}$ identified that serum exosomal miR-146a-5p might be a new biomarker for predicting the efficacy of DDP for NSCLC patients and real-time monitoring drug resistance. Shi et al. ${ }^{[67]}$ also investigated the role of miR-146a in the development of acquired drug resistance to DDP in NSCLC cells, and found that overexpression of miR-146a significantly increased the sensitivity of NSCLC cells to DDP via blocking the cell cycle, enhancing cell apoptosis, and inhibiting cell viability and motility both in vitro and in vivo. Ma et al. ${ }^{[68]}$ reported that DLK1 was a potential target for miR-129-5p, and overexpression of miR-129-5p could inhibit NSCLC chemo-resistance to DDP through regulating DLK1. Li et al. ${ }^{[69]}$ found that miR-196a was associated with the development of NSCLC. Down-regulation of miR-196a may increase the sensitivity of NSCLC cells to DDP by inducing apoptosis in vitro and in vivo. Another study ${ }^{[70]}$ indicated that miR-181c conferred resistance to DDP in NSCLC cells by activating the Wnt/ $\beta$-catenin pathway through inhibiting WIF1, providing a potential therapeutic target for NSCLC patients with DDP resistance.

In summary, these studies suggest that miRNAs are closely related to the resistance of NSCLC to radiotherapy and chemotherapy, but research into the rela- tionship between miRNAs and therapeutic resistance is still in its infancy. Before applying this theory in clinical practice, studies with larger sample sizes and methods validation procedures will be indispensable.

\section{CONCLUSION}

The abnormal expression of miRNAs is closely related to the occurrence and development of NSCLC and regulates the differentiation, proliferation and apoptosis of NSCLC cells, participates in the invasion and metastasis of NSCLC cells, and affects resistance to anti-cancer drugs. Additionally, miRNAs have potential value in the diagnosis, treatment, prognosis and risk stratification of NSCLC. However, the application of miRNAs is mainly based on laboratory research, which lacks large-scale clinical transformation research. Therefore, a more in-depth study of miRNAs may lead to a new method for the diagnosis and treatment of lung cancer.

\section{REFERENCES}

1. Ferlay, J., Soerjomataram, I., Dikshit, R., Eser, S., Mathers, C., Rebelo, M., Parkin, D. M., Forman, D., and Bray, F. (2015) Cancer incidence and mortality worldwide: sources, methods and major patterns in GLOBOCAN 2012. International journal of cancer 136, E359-386

2. Lu, Y. Q., and Lu, K. H. (2017) Advancements in next-generation sequencing for diagnosis and treatment of nonsmall-cell lung cancer. Chronic diseases and translational medicine $3,1-7$

3. Wang, L., Yu, C., Liu, Y., Wang, J., Li, C., Wang, Q., Wang, P., Wu, S., and Zhang, Z. J. (2016) Lung Cancer Mortality Trends in China from 1988 to 2013: New Challenges and Opportunities for the Government. International journal of environmental research and public health 13 , E1052

4. Siegel, R. L., Miller, K. D., and Jemal, A. (2017) Cancer Statistics, 2017. CA: a cancer journal for clinicians 67, 7-30

5. Allemani, C., Weir, H. K., Carreira, H., Harewood, R., Spika, D., Wang, X. S., Bannon, F., Ahn, J. V., Johnson, C. J., Bonaventure, A., Marcos-Gragera, R., Stiller, C., Azevedo e Silva, G., Chen, W. Q., Ogunbiyi, O. J., Rachet, B., Soeberg, M. J., You, H., Matsuda, T., Bielska-Lasota, M., Storm, H., Tucker, T. C., and Coleman, M. P. (2015) Global surveillance of cancer survival 1995-2009: analysis of individual data for $25,676,887$ patients from 279 population-based registries in 67 countries (CONCORD-2). Lancet (London, England) 385, 977-1010

6. Takasaki, S. (2015) Roles of microRNAs in cancers and development. Methods in molecular biology (Clifton, N.J.) 1218, 375-413

7. Keshavarzi, M., Sorayayi, S., Jafar Rezaei, M., Mohammadi, M., Ghaderi, A., Rostamzadeh, A., Masoudifar, 
A., and Mirzaei, H. (2017) MicroRNAs-Based Imaging Techniques in Cancer Diagnosis and Therapy. Journal of cellular biochemistry 118, 4121-4128

8. Pastorkova, Z., Skarda, J., and Andel, J. (2016) The role of microRNA in metastatic processes of non-small cell lung carcinoma. Biomedical papers of the Medical Faculty of the University Palacky, Olomouc, Czechoslovakia 160, 343-357

9. Skrzypski, M., Dziadziuszko, R., and Jassem, J. (2011) MicroRNA in lung cancer diagnostics and treatment. Mutation research 717, 25-31

10. Ma, R., Wang, C., Wang, J., Wang, D., and Xu, J. (2016) miRNA-mRNA Interaction Network in Non-small Cell Lung Cancer. Interdisciplinary sciences, computational life sciences 8, 209-219

11. Zhou, Q., Huang, S. X., Zhang, F., Li, S. J., Liu, C., Xi, Y. Y., Wang, L., Wang, X., He, Q. Q., Sun, C. C., and Li, D. J. (2017) MicroRNAs: A novel potential biomarker for diagnosis and therapy in patients with non-small cell lung cancer. Cell proliferation 50, e12394

12. Yin, Z., Xu, M., and Li, P. (2017) miRNA-221 acts as an oncogenic role by directly targeting TIMP2 in nonsmall-cell lung carcinoma. Gene 620, 46-53

13. Zhou, S., Zhang, Z., Zheng, P., Zhao, W., and Han, N. (2017) MicroRNA-1285-5p influences the proliferation and metastasis of non-small-cell lung carcinoma cells via downregulating CDH1 and Smad4. Tumour biology : the journal of the International Society for Oncodevelopmental Biology and Medicine 39, 1010428317705513

14. Wang, Y., Li, Y., Wu, B., Shi, C., and Li, C. (2017) MicroRNA-661 promotes non-small cell lung cancer progression by directly targeting RUNX3. Molecular medicine reports 16, 2113-2120

15. Liu, F., Cai, Y., Rong, X., Chen, J., Zheng, D., Chen, L., Zhang, J., Luo, R., Zhao, P., and Ruan, J. (2017) MiR-661 promotes tumor invasion and metastasis by directly inhibiting RB1 in non small cell lung cancer. Molecular cancer 16,122

16. Huang, J., Sun, C., Wang, S., He, Q., and Li, D. (2015) microRNA miR-10b inhibition reduces cell proliferation and promotes apoptosis in non-small cell lung cancer (NSCLC) cells. Molecular bioSystems 11, 2051-2059

17. Li, T., Ding, Z. L., Zheng, Y. L., and Wang, W. (2017) MiR484 promotes non-small-cell lung cancer (NSCLC) progression through inhibiting Apaf-1 associated with the suppression of apoptosis. Biomedicine \& pharmacotherapy = Biomedecine \& pharmacotherapie 96, 153-164

18. Baumgartner, U., Berger, F., Hashemi Gheinani, A., Burgener, S. S., Monastyrskaya, K., and Vassella, E. (2018) miR$19 \mathrm{~b}$ enhances proliferation and apoptosis resistance via the EGFR signaling pathway by targeting PP2A and BIM in non-small cell lung cancer. Molecular cancer 17, 44

19. Zeng, Y., Zhu, J., Shen, D., Qin, H., Lei, Z., Li, W., Liu, Z., and Huang, J. A. (2017) MicroRNA-205 targets SMAD4 in non-small cell lung cancer and promotes lung cancer cell growth in vitro and in vivo. Oncotarget 8, 30817-30829

20. Sun, C. C., Li, S. J., Yuan, Z. P., and Li, D. J. (2016) MicroRNA-346 facilitates cell growth and metastasis, and suppresses cell apoptosis in human non-small cell lung cancer by regulation of XPC/ERK/Snail/E-cadherin pathway. Aging 8, 2509-2524

21. Bu, W., and Luo, T. (2017) miR-1297 Promotes Cell Proliferation of Non-Small Cell Lung Cancer Cells: Involving in PTEN/Akt/Skp2 Signaling Pathway. DNA and cell biology 36, 976-982

22. Xie, X., Liu, H. T., Mei, J., Ding, F. B., Xiao, H. B., Hu, F. Q., Hu, R., and Wang, M. S. (2015) miR-106a promotes growth and metastasis of non-small cell lung cancer by targeting PTEN. International journal of clinical and experimental pathology 8, 3827-3834

23. Wang, Y., Cong, W., Wu, G., Ju, X., Li, Z., Duan, X., Wang, X., and Gao, H. (2018) MiR-376a suppresses the proliferation and invasion of non-small-cell lung cancer by targeting c-Myc. Cell biology international 42, 25-33

24. Yang, C. L., Zheng, X. L., Ye, K., Ge, H., Sun, Y. N., Lu, Y. F., and Fan, Q. X. (2018) MicroRNA-183 Acts as a Tumor Suppressor in Human Non-Small Cell Lung Cancer by Down-Regulating MTA1. Cellular physiology and biochemistry : international journal of experimental cellular physiology, biochemistry, and pharmacology 46, 93-106

25. Xiao, P., and Liu, W. L. (2015) MiR-142-3p functions as a potential tumor suppressor directly targeting HMGB1 in non-small-cell lung carcinoma. International journal of clinical and experimental pathology 8, 10800-10807

26. Wang, P., Lv, H. Y., Zhou, D. M., and Zhang, E. N. (2016) miR-204 suppresses non-small-cell lung carcinoma (NSCLC) invasion and migration by targeting JAK2. Genetics and molecular research : GMR 15, gmr6415

27. Zhang, S., Gao, L., Thakur, A., Shi, P., Liu, F., Feng, J., Wang, T., Liang, Y., Liu, J. J., Chen, M., and Ren, H. (2016) miRNA-204 suppresses human non-small cell lung cancer by targeting ATF2. Tumour biology : the journal of the International Society for Oncodevelopmental Biology and Medicine 37, 11177-11186

28. Zhang, Z., Yang, Y., and Zhang, X. (2017) MiR-770 inhibits tumorigenesis and EMT by targeting JMJD6 and regulating WNT/beta-catenin pathway in non-small cell lung cancer. Life sciences 188, 163-171

29. Chen, G. M., Zheng, A. J., Cai, J., Han, P., Ji, H. B., and Wang, L. L. (2018) microRNA-145-3p inhibits non-small cell lung cancer cell migration and invasion by targeting PDK1 via the mTOR signaling pathway. Journal of cellular biochemistry 119, 885-895

30. Zhou, Y., Li, S., Li, J., Wang, D., and Li, Q. (2017) Effect of microRNA-135a on Cell Proliferation, Migration, Invasion, Apoptosis and Tumor Angiogenesis Through the IGF-1/PI3K/Akt Signaling Pathway in Non-Small Cell Lung Cancer. Cellular physiology and biochemistry : in- 
ternational journal of experimental cellular physiology, biochemistry, and pharmacology 42, 1431-1446

31. Jiang, W., Wei, K., Pan, C., Li, H., Cao, J., Han, X., Tang, Y., Zhu, S., Yuan, W., He, Y., Xia, Y., Chen, L., and Chen, Y. (2018) MicroRNA-1258 suppresses tumour progression via GRB2/Ras/Erk pathway in non-small-cell lung cancer. Cell proliferation 2018, e12502

32. Liu, B., Li, J., Zheng, M., Ge, J., Li, J., and Yu, P. (2017) MiR542-3p exerts tumor suppressive functions in non-small cell lung cancer cells by upregulating FTSJ2. Life sciences 188, 87-95

33. Ma, N., Zhang, W., Qiao, C., Luo, H., Zhang, X., Liu, D., Zang, S., Zhang, L., and Bai, J. (2016) The Tumor Suppressive Role of MiRNA-509-5p by Targeting FOXM1 in Non-Small Cell Lung Cancer. Cellular physiology and biochemistry : international journal of experimental cellular physiology, biochemistry, and pharmacology 38, 1435-1446

34. Mi, J., Zou, Y., Lin, X., Lu, J., Liu, X., Zhao, H., Ye, X., Hu, H., Jiang, B., Han, B., Shao, C., and Gong, Y. (2017) Dysregulation of the miR-194-CUL4B negative feedback loop drives tumorigenesis in non-small-cell lung carcinoma. Molecular oncology 11, 305-319

35. Zhao, X., Lu, C., Chu, W., Zhang, B., Zhen, Q., Wang, R., Zhang, Y., Li, Z., Lv, B., Li, H., and Liu, J. (2017) MicroRNA-124 suppresses proliferation and glycolysis in nonsmall cell lung cancer cells by targeting AKT-GLUT1/ HKII. Tumour biology : the journal of the International Society for Oncodevelopmental Biology and Medicine 39, 1010428317706215

36. Kerbel, R. S. (2000) Tumor angiogenesis: past, present and the near future. Carcinogenesis 21, 505-515

37. Hu, J., Cheng, Y., Li, Y., Jin, Z., Pan, Y., Liu, G., Fu, S., Zhang, Y., Feng, K., and Feng, Y. (2014) microRNA-128 plays a critical role in human non-small cell lung cancer tumourigenesis, angiogenesis and lymphangiogenesis by directly targeting vascular endothelial growth factor-C. European journal of cancer (Oxford, England : 1990) 50, 2336-2350

38. Switlik, W., Karbownik, M. S., Suwalski, M., Kozak, J., and Szemraj, J. (2018) miR-30a-5p together with miR-210$3 p$ as a promising biomarker for non-small cell lung cancer: A preliminary study. Cancer biomarkers : section A of Disease markers 21, 479-488

39. Geng, Q., Fan, T., Zhang, B., Wang, W., Xu, Y., and Hu, H. (2014) Five microRNAs in plasma as novel biomarkers for screening of early-stage non-small cell lung cancer. Respiratory research 15, 149

40. Zhu, W., Zhou, K., Zha, Y., Chen, D., He, J., Ma, H., Liu, X., Le, H., and Zhang, Y. (2016) Diagnostic Value of Serum miR-182, miR-183, miR-210, and miR-126 Levels in Patients with Early-Stage Non-Small Cell Lung Cancer. PloS one 11, e0153046

41. Jin, X., Chen, Y., Chen, H., Fei, S., Chen, D., Cai, X., Liu, L., Lin, B., Su, H., Zhao, L., Su, M., Pan, H., Shen, L., Xie, D., and Xie, C. (2017) Evaluation of Tumor-Derived Exosomal miRNA as Potential Diagnostic Biomarkers for Early-Stage Non-Small Cell Lung Cancer Using Next-Generation Sequencing. Clinical cancer research : an official journal of the American Association for Cancer Research 23, 5311-5319

42. Molina-Pinelo, S., Gutierrez, G., Pastor, M. D., Hergueta, M., Moreno-Bueno, G., Garcia-Carbonero, R., Nogal, A., Suarez, R., Salinas, A., Pozo-Rodriguez, F., Lopez-Rios, F., Agullo-Ortuno, M. T., Ferrer, I., Perpina, A., Palacios, J., Carnero, A., and Paz-Ares, L. (2014) MicroRNA-dependent regulation of transcription in non-small cell lung cancer. PloS one 9, e90524

43. Wang, C., Ding, M., Xia, M., Chen, S., Van Le, A., Soto-Gil, R., Shen, Y., Wang, N., Wang, J., Gu, W., Wang, X., Zhang, Y., Zen, K., Chen, X., Zhang, C., and Zhang, C. Y. (2015) A Five-miRNA Panel Identified From a Multicentric Case-control Study Serves as a Novel Diagnostic Tool for Ethnically Diverse Non-small-cell Lung Cancer Patients. EBioMedicine 2, 1377-1385

44. Giallombardo, M., Chacartegui Borras, J., Castiglia, M., Van Der Steen, N., Mertens, I., Pauwels, P., Peeters, M., and Rolfo, C. (2016) Exosomal miRNA Analysis in Non-small Cell Lung Cancer (NSCLC) Patients' Plasma Through qPCR: A Feasible Liquid Biopsy Tool. Journal of visualized experiments : JoVE 2016, e53900

45. Zhan, B., Lu, D., Luo, P., and Wang, B. (2016) Prognostic Value of Expression of MicroRNAs in Non-Small Cell Lung Cancer: A Systematic Review and Meta-Analysis. Clinical laboratory 62, 2203-2211

46. Wang, K., Dong, L., Fang, Q., Xia, H., and Hou, X. (2017) Low serum miR-98 as an unfavorable prognostic biomarker in patients with non-small cell lung cancer. Cancer biomarkers : section A of Disease markers 20, 283288

47. Guo, W., Zhang, Y., Zhang, Y., Shi, Y., Xi, J., Fan, H., and Xu, S. (2015) Decreased expression of miR-204 in plasma is associated with a poor prognosis in patients with nonsmall cell lung cancer. International journal of molecular medicine 36, 1720-1726

48. Wang, S. Y., Li, Y., Jiang, Y. S., and Li, R. Z. (2017) Investigation of serum miR-411 as a diagnosis and prognosis biomarker for non-small cell lung cancer. European review for medical and pharmacological sciences 21 , 4092-4097

49. Li, J., Yang, H., Li, Y., Liu, Y., Chen, S., Qi, C., Zhang, Q., Lan, T., He, X., Guan, X. Y., and Wang, L. (2014) microRNA-146 up-regulation predicts the prognosis of non-small cell lung cancer by miRNA in situ hybridization. Experimental and molecular pathology 96, 195-199

50. Zhang, Y., Zhao, Y., Sun, S., Liu, Z., Zhang, Y., and Jiao, S. (2016) Overexpression of MicroRNA-221 is associated with poor prognosis in non-small cell lung cancer patients. Tumour biology : the journal of the International 
Society for Oncodevelopmental Biology and Medicine 37, 10155-10160

51. Hale, M. D., Hayden, J. D., and Grabsch, H. I. (2013) Tumour-microenvironment interactions: role of tumour stroma and proteins produced by cancer-associated fibroblasts in chemotherapy response. Cellular oncology (Dordrecht) 36, 95-112

52. Chen, Y., Du, M., Wang, J., Xing, P., Zhang, Y., Li, F., and Lu, X. (2016) MiRNA-200a expression is inverse correlation with hepatocyte growth factor expression in stromal fibroblasts and its high expression predicts a good prognosis in patients with non-small cell lung cancer. Oncotarget 7, 48432-48442

53. Zhao, Y., Wei, Q., Hu, L., Chen, F., Hu, Z., Heist, R. S., Su, L., Amos, C. I., Shen, H., and Christiani, D. C. (2014) Polymorphisms in MicroRNAs are associated with survival in non-small cell lung cancer. Cancer epidemiology, biomarkers \& prevention : a publication of the American Association for Cancer Research, cosponsored by the American Society of Preventive Oncology 23, 25032511

54. Liu, Q., Yu, Z., Yuan, S., Xie, W., Li, C., Hu, Z., Xiang, Y., Wu, N., Wu, L., Bai, L., and Li, Y. (2017) Circulating exosomal microRNAs as prognostic biomarkers for non-small-cell lung cancer. Oncotarget 8, 13048-13058

55. Kanaoka, R., Iinuma, H., Dejima, H., Sakai, T., Uehara, H., Matsutani, N., and Kawamura, M. (2018) Usefulness of Plasma Exosomal MicroRNA-451a as a Noninvasive Biomarker for Early Prediction of Recurrence and Prognosis of Non-Small Cell Lung Cancer. Oncology 94, 311323

56. Salim, H., Akbar, N. S., Zong, D., Vaculova, A. H., Lewensohn, R., Moshfegh, A., Viktorsson, K., and Zhivotovsky, B. (2012) miRNA-214 modulates radiotherapy response of non-small cell lung cancer cells through regulation of p38MAPK, apoptosis and senescence. British journal of cancer 107, 1361-1373

57. Ma, W., Ma, C. N., Li, X. D., and Zhang, Y. J. (2016) Examining the effect of gene reduction in miR-95 and enhanced radiosensitivity in non-small cell lung cancer. Cancer gene therapy 23, 66-71

58. Yin, H., Ma, J., Chen, L., Piao, S., Zhang, Y., Zhang, S., Ma, H., Li, Y., Qu, Y., Wang, X., and Xu, Q. (2018) MiR-99a Enhances the Radiation Sensitivity of Non-Small Cell Lung Cancer by Targeting mTOR. Cellular physiology and biochemistry : international journal of experimental cellular physiology, biochemistry, and pharmacology 46, 471-481

59. Wang, X. C., Wang, W., Zhang, Z. B., Zhao, J., Tan, X. G., and Luo, J. C. (2013) Overexpression of miRNA-21 promotes radiation-resistance of non-small cell lung cancer. Radiation oncology (London, England) 8, 146
60. Ma, W., Ma, C. N., Zhou, N. N., Li, X. D., and Zhang, Y. J. (2016) Up- regulation of miR-328-3p sensitizes nonsmall cell lung cancer to radiotherapy. Scientific reports 6, 31651

61. Chen, X., Xu, Y., Liao, X., Liao, R., Zhang, L., Niu, K., Li, T., Li, D., Chen, Z., Duan, Y., and Sun, J. (2016) Plasma miRNAs in predicting radiosensitivity in non-small cell lung cancer. Tumour biology : the journal of the International Society for Oncodevelopmental Biology and Medicine 37, 11927-11936

62. Li, L., Li, C., Wang, S., Wang, Z., Jiang, J., Wang, W., Li, X., Chen, J., Liu, K., Li, C., and Zhu, G. (2016) Exosomes Derived from Hypoxic Oral Squamous Cell Carcinoma Cells Deliver miR-21 to Normoxic Cells to Elicit a Prometastatic Phenotype. Cancer research 76, 1770-1780

63. Ye, J., Zhang, Z., Sun, L., Fang, Y., Xu, X., and Zhou, G. (2016) miR-186 regulates chemo-sensitivity to paclitaxel via targeting MAPT in non-small cell lung cancer (NSCLC). Molecular bioSystems 12, 3417-3424

64. Xu, X., Jin, S., Ma, Y., Fan, Z., Yan, Z., Li, W., Song, Q., You, W., Lyu, Z., Song, Y., Shi, P., Liu, Y., Han, X., Li, L., Li, Y., Liu, Y., and Ye, Q. (2017) miR-30a-5p enhances paclitaxel sensitivity in non-small cell lung cancer through targeting BCL-2 expression. Journal of molecular medicine (Berlin, Germany) 95, 861-871

65. Gan, C. Z., Li, G., Luo, Q. S., and Li, H. M. (2017) miR-339$5 p$ downregulation contributes to Taxol resistance in small-cell lung cancer by targeting alpha1,2-fucosyltransferase 1. IUBMB life 69, 841-849

66. Yuwen, D. L., Sheng, B. B., Liu, J., Wenyu, W., and Shu, Y. Q. (2017) MiR-146a-5p level in serum exosomes predicts therapeutic effect of cisplatin in non-small cell lung cancer. European review for medical and pharmacological sciences 21, 2650-2658

67. Shi, L., Xu, Z., Wu, G., Chen, X., Huang, Y., Wang, Y., Jiang, W., and Ke, B. (2017) Up-regulation of miR-146a increases the sensitivity of non-small cell lung cancer to DDP by downregulating cyclin J. BMC cancer 17, 138

68. Ma, Z., Cai, H., Zhang, Y., Chang, L., and Cui, Y. (2017) MiR129-5p inhibits non-small cell lung cancer cell stemness and chemoresistance through targeting DLK1. Biochemical and biophysical research communications 490, 309316

69. Li, Q., Yang, Z., Chen, M., and Liu, Y. (2016) Downregulation of microRNA-196a enhances the sensitivity of nonsmall cell lung cancer cells to cisplatin treatment. International journal of molecular medicine 37, 1067-1074

70. Zhang, H., Hu, B., Wang, Z., Zhang, F., Wei, H., and Li, L. (2017) miR-181c contributes to cisplatin resistance in non-small cell lung cancer cells by targeting Wnt inhibition factor 1 . Cancer chemotherapy and pharmacology $80,973-984$ 